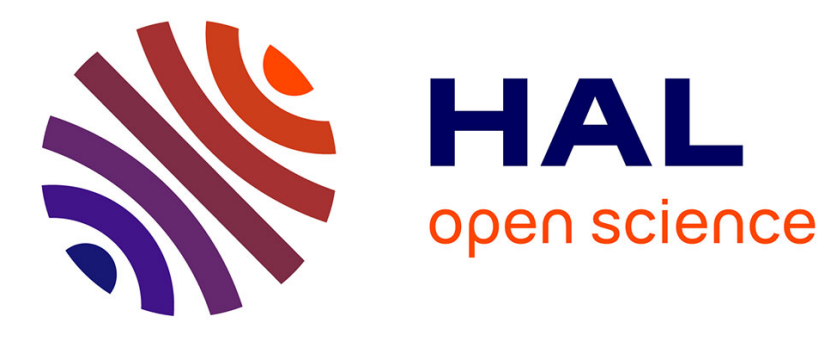

\title{
Damage Detection with Auxiliary Subsystem
}

Fabrizio Vestroni, Jacopo Ciambella, Francesco Dell'Isola, Stefano Vidoli

\section{To cite this version:}

Fabrizio Vestroni, Jacopo Ciambella, Francesco Dell'Isola, Stefano Vidoli. Damage Detection with Auxiliary Subsystem. CIMTEC 2008 - Proceedings of 3rd International Conference on Smart Materials, Structures and Systems - Emboding Intelligence in Structures and Integrated Systems, 2008, pp.15. hal-00502461

\section{HAL Id: hal-00502461 \\ https://hal.science/hal-00502461}

Submitted on 15 Jul 2010

HAL is a multi-disciplinary open access archive for the deposit and dissemination of scientific research documents, whether they are published or not. The documents may come from teaching and research institutions in France or abroad, or from public or private research centers.
L'archive ouverte pluridisciplinaire HAL, est destinée au dépôt et à la diffusion de documents scientifiques de niveau recherche, publiés ou non, émanant des établissements d'enseignement et de recherche français ou étrangers, des laboratoires publics ou privés. 


\title{
Damage Detection with Auxiliary Subsystem
}

\author{
F. Vestroni, J. Ciambella, F. dell'Isola, S. Vidoli
}

April 26, 2008

\section{Contents}

1 Introduction 1

2 System description $\quad 2$

2.1 Auxiliary subsystems and measured frequency responses . . . . . 2

3 Localization technique $\mathbf{5}$

3.1 Parametrization of stiffness distribution and damage functional . 5

3.2 Model independent pre-localization technique . . . . . . . . . . 7

4 Numerical Examples $\quad 9$

5 Conclusions $\quad 9$

A Proof of main results 11

A.1 Independence of the damage functional on the shape . . . . . . . 11

\section{Introduction}

Among Non-Destructive Evaluation, vibration-based methods present some peculiarities. Structural damages change the dynamic behavior of the system, and vibrations should be used to detect these changes and to evaluate damages placed not accessible locations.

These methods respond to the current tendency which is to integrate into the structure identification and monitoring systems; to this aim transducers and sensors are embedded with the mechanical system and driven by suitably designed electronic controllers.

The major limit of this and the other indirect identification techniques based on frequency response measurements turns out to be the small sensitivity to local variations of mechanical characteristics: it reflects on a uncertainty on the identified parameters, since it implies a small curvature of any reasonable identification functional build on these quantities.

An enlightening example for the application of the proposed technique is given through a beam-like structure coupled to a network of piezoelectric patches. 
Although the forces exerted by the piezoelectric transducers are not large, the choice of such a kind of actuators/sensors implies the remarkable advantage of dealing with highly sensitive and easily tunable devices.

The proposed damage identification procedure allows an effective damage estimation -both position and intensity are estimated- through a suitable electronic circuit. Under some hypothesis on the spatial distribution of the transducers, the procedure allowing for the damage localization does not rely on the model of the mechanical subsystem and, hence, could be applied to different structural members.

\section{System description}

\subsection{Auxiliary subsystems and measured frequency responses}

Following [3] in the next it is assumed that the overall system is governed by the equations of motion:

$$
\left\{\begin{array}{l}
{\left[k(x) w^{\prime \prime}(x, t)\right]^{\prime \prime}+\rho(x) \ddot{w}(x, t)+c \rho(x) \dot{w}(x, t)+\sum_{i=1}^{N} g_{i} \dot{\psi}_{i} p_{i}^{\prime \prime}(x)=f(x, t),} \\
Y_{i j} \psi_{j}(t)+R_{i j} \dot{\psi}_{j}(t)+B_{i j} \ddot{\psi}_{j}(t)-g_{i} \int_{0}^{\ell} \dot{w}(x, t) p_{i}^{\prime \prime}(x) d x=q_{i}(t),
\end{array}\right.
$$

and initial conditions:

$$
\left\{\begin{array}{l}
w(\cdot, 0)=w_{0}, \dot{w}(\cdot, 0)=\dot{w}_{0} \\
\dot{\psi}_{j}(0)=\psi_{j 0}, \dot{\psi}_{j}(0)=\chi_{j 0}
\end{array}\right.
$$

The structural model assumed here is a simple 1-D Euler-Bernoulli like for both the beam and the attached piezoelectric elements; therefore the scalar field $w(x, t)$ represents the transverse deflection of the beam axis and $\psi_{j}, i=$ $1, \ldots, N$ is the vector of nodal flux linkages at the terminal of the $N$ piezoelectric transducers.

In equation (2), as usual, a superimposed dot represents a time derivative while a prime is the derivative with respect to the spacial variable $x$.

The functions $p_{i}(x)$ for $i=1,2, \ldots N$ are the characteristic functions of the piezoelectric supports:

$$
p_{i}(x):=\left\{\begin{array}{l}
1 x \in i-\text { th PZT region } \\
0 \text { otherwise }
\end{array}\right.
$$

and therefore their derivatives with respect to the abscissa $x$ must be understood in a distributional sense. The additional current due to electromechanical coupling is computed as follows:

$$
q_{i}^{C}(t)=-g_{i} \int_{0}^{\ell} \dot{w}^{\prime \prime}(x, t) p_{i}(x) d x=-g_{i} \ell_{i} \overline{\left.\dot{w}^{\prime \prime}\right|_{i}}
$$


where $l_{i}$ is the width of the $i$-th PZT patch.

A finite dimensional model for the overall system may be obtained by a suitable projection of the displacement field:

$$
w(x, t):=\sum_{\alpha=1}^{A} \phi_{\alpha}(x) W_{\alpha}(t)
$$

where $\phi_{\alpha} \in C^{2}(\mathbb{R})$ are the shape functions. From equation (1) one gets:

$$
\begin{gathered}
\left\{\begin{array}{l}
K_{\alpha \beta}(\pi) W_{\beta}(t)+C_{\alpha \beta} \dot{W}_{\beta}(t)+M_{\alpha \beta} \ddot{W}_{\beta}(t)+\Gamma_{\alpha i} \dot{\psi}_{i}(t)=F_{\alpha}(t), \\
Y_{i j} \psi_{j}(t)+R_{i j} \dot{\psi}_{j}(t)+B_{i j} \ddot{\psi}_{j}(t)-\Gamma_{\alpha i} \dot{W}_{\alpha}(t)=q_{i}(t),
\end{array}\right. \\
\left\{\begin{array}{l}
W_{\beta}(0)=W_{\beta 0}, \dot{W}_{\beta}(0)=V_{\beta 0}, \\
\dot{\psi}_{j}(0)=\psi_{j 0}, \dot{\psi}_{j}(0)=\chi_{j 0},
\end{array}\right.
\end{gathered}
$$

where $j=1, \ldots, N, \alpha=1, \ldots, A, \beta=1, \ldots, A$ and

$$
\begin{aligned}
K_{\alpha \beta}(\pi) & :=\int_{0}^{\ell} k(\pi ; x) \phi_{\alpha}^{\prime \prime}(x) \phi_{\beta}^{\prime \prime}(x) d x \\
\Gamma_{\alpha i} & :=g_{i} \int_{0}^{\ell} p_{i}^{\prime \prime}(x) \phi_{\alpha}(x) d x=g_{i} \int_{0}^{\ell} p_{i}(x) \phi_{\alpha}^{\prime \prime}(x) d x, \\
M_{\alpha \beta} & :=\int_{0}^{\ell} \rho(x) \phi_{\alpha}(x) \phi_{\beta}(x) d x, \quad C_{\alpha \beta}:=c M_{\alpha \beta}, \\
F(t) & :=\int_{0}^{\ell} f(x, t) \phi_{\alpha}(x) d x .
\end{aligned}
$$

Here $W_{\alpha}$ and $\psi_{j}$ represent the degrees of freedom of the given structure and the additional dynamical system respectively. As a consequence $K$ and $Y, C$ and $R, M$ and $B$ can be interpreted as the stiffness, damping and mass matrices for the two subsystems, whilst $F$ and $Q$ are the external applied actions. The matrix $\Gamma$ models the gyroscopic coupling between the structure and the additional subsystem. The structural stiffness matrix $K$ is assumed to depend on the set of monitored parameters $\pi \in \Pi \subset \mathbb{R}^{P}$.

The Laplace transform of (2.1) leads to:

$$
\left\{\begin{array}{l}
{\left[K_{\alpha \beta}(\pi)+s C_{\alpha \beta}+s^{2} M_{\alpha \beta}\right] \tilde{W}_{\beta}(s)+s \Gamma_{\alpha i} \tilde{\psi}_{i}(s)=\bar{F}_{\alpha}(s),} \\
{\left[Y_{i j}+s R_{i j}+s^{2} B_{i j}\right] \tilde{\psi}_{j}(s)-s \Gamma_{\alpha i} \tilde{W}_{\alpha}(s)=\bar{q}_{i}(s),}
\end{array}\right.
$$

where

$$
\left\{\begin{array}{l}
\bar{F}_{\alpha}(s):=\tilde{F}_{\alpha}(s)+\left(C_{\alpha \beta}+s M_{\alpha \beta}\right) W_{\beta 0}+M_{\alpha \beta} V_{\beta 0}+\Gamma_{\alpha i} \psi_{i 0} \\
\bar{q}_{i}(s):=\tilde{q}_{i}(s)+\left(R_{i j}+s B_{i j}\right) \psi_{j 0}+B_{i j} \chi_{j 0}-\Gamma_{\alpha i} W_{\alpha 0}
\end{array}\right.
$$


and a superimposed tilde means the Laplace transform of the corresponding time function, and $s$ is the Laplace variable.

Solving $(9)_{1}$ for the vector $\tilde{W}$ and replacing in $(9)_{2}$ one gets:

$$
\left[\left(Y_{i j}+s R_{i j}+s^{2} B_{i j}\right)+s^{2} \Gamma_{\alpha i} H_{\alpha \beta}(s, \pi) \Gamma_{\beta j}\right] \tilde{\psi}_{j}=\bar{q}_{i}-s \Gamma_{\alpha i} \bar{F}_{\alpha} .
$$

where $H(s, \pi):=\left[K(\pi)+s C+s^{2} M\right]^{-1}$ is the frequency response matrix of the mechanical sub-structure. The algebraic manipulation performed was aimed to establish an explicit relationship between the additional degrees of freedom $\tilde{\psi}$ and their dual quantities $\tilde{q}$; both of them are supposed to be easily measurable and/or imposable: in the instance considered below this is indeed the case, they represent electric flux linkages and currents.

Consideration of equation (10) for vanishing initial conditions and mechanical applied forces, i.e. when $W_{\alpha 0}=\tilde{F}_{\alpha}=V_{\alpha 0}=0$ and $\psi_{i 0}=\chi_{i 0}=0$, allows for the definition of two mutually inverse frequency response functions $(F R F)$ :

$$
\begin{aligned}
N_{i j}(s, \pi) \tilde{\psi}_{j}=\tilde{q}_{i}, & N_{i j}(s \pi):=Y_{i j}+s R_{i j}+s^{2} B_{i j}+s^{2} \Gamma_{\alpha i} H_{\alpha \beta}(s, \pi) \Gamma_{\beta j} . \\
T_{i j}(s, \pi) \tilde{q}_{j}=\tilde{\psi}_{i}, & T_{i j}(s \pi):=\left[\left(Y+s R+s^{2} B+s^{2} \Gamma H(s, \pi) \Gamma\right)^{-1}\right]_{i j} .
\end{aligned}
$$

The $N_{i j}(s)$ FRF asks for an electronic interface circuit devoted to write the currents on each PZT patches whilst measuring their voltages. Moreover this FRF enjoys the useful feature of being representable as the sum of the purely electric admittance constant with respect to the parameters $\pi$, plus the function:

$$
\mathfrak{H}_{i j}(s, \pi):=s^{2} \Gamma_{\alpha i} H_{\alpha \beta}(s, \pi) \Gamma_{\beta j},
$$

which can be physically interpreted as the additional admittance seen in the auxiliary subsystem because of its coupling with the preexisting structure.

Concerning the additional mechanical impedance $\mathfrak{H}(s, \pi)$ we remark that:

- its experimental determination is obtained as a difference between the frequency response $N_{i j}(s, \pi)$ and the purely electric impedance $N_{0}(s)=$ $Y+s R+s^{2} B$.

- is independent on the electric parameters $Y, R$ and $B$; as this its sensitivity can not be affected (in particular enhanced) acting on this parameters.

- it contains information only on the mechanical eigenfrequencies; actually these are the poles of the function $\mathfrak{H}(i \omega, \pi)$ with respect to the pulsation $\omega$.

- it contains information on the mechanical eigenvectors as sampled and filtered by the coupling matrix $\Gamma$.

More precisely, using the spectral decomposition of the mechanical frequency response function:

$$
H_{\alpha \beta}(s, \pi):=\sum_{h=1}^{\infty} \frac{u_{\alpha}^{(h)} u_{\beta}^{(h)}}{s^{2}+\varpi_{h}^{2}+2 s \delta_{h} \varpi_{h}},
$$


in terms of mechanical eigenvectors $\left\{u^{(h)}\right\}_{h=1,2 \ldots}$ and eigenfrequencies $\left\{\varpi_{h}\right\}_{h=1,2 \ldots}$, one obtains:

$$
\mathfrak{H}_{i j}(s, \pi)=s^{2} \sum_{h=1}^{\infty} \frac{z_{i}^{(h)} z_{j}^{(h)}}{s^{2}+\varpi_{h}^{2}+2 s \delta_{h} \varpi_{h}}, \quad z_{i}^{(h)}:=\sum_{\alpha} \Gamma_{\alpha i} u_{\alpha}^{(h)} .
$$

On the contrary the dependance of the $\mathrm{FRF} T_{i j}$ on the constitutive matrices $Y, R$ and $B$ of the additional subsystem, may be exploited - at the cost of considering more involved relationships - to get a sensitivity enhancement in damage detection. But this problem will be addressed in a future work.

Here the attention is instead focused on a damage identification procedure taking advantage of the discussed properties of $\mathfrak{H}$.

\section{Localization technique}

\subsection{Parametrization of stiffness distribution and damage functional}

In order to represent the stiffness variations on the structural member the following procedure is used:

- the whole domain is decomposed as the union of $H$ macro-regions; such a decomposition is induced by the actual partitioning of the PZT patches;

- a piecewise linear interpolation is performed for the bending stiffness function using the centres of mass $\left(G_{h}\right)$ of these macro-regions.

In particular when the domain is monodimensional, as in the examined system, the bending stiffness $k(x)$ can be expressed as follows:

$$
k(x)=k_{0}(x)+\sum_{h=1}^{H} d_{h} f_{h}\left(G_{h}, b_{h} ; x\right)=k_{0}(x)+\Delta k(\pi, x)
$$

where $f_{h}\left(G_{h}, b_{h} ; x\right)$ is a function which is 1 in the center of mass of the $h$-th macro-region and is 0 outside that macro-region, that is:

$$
f_{h}\left(G_{h}, b_{h} ; x\right)=\left\{\begin{array}{c}
2 \frac{x-G_{h}}{b_{h}}+1, \quad G_{h}-b_{h} / 2 \leq x \leq G_{h} \\
1-2 \frac{G_{h}-x}{b_{h}}, \quad G_{h} \leq x \leq G_{h}+b_{h} / 2 \\
0, \quad x \leq G_{h}-b_{h} / 2, \quad G_{h}+b_{h} / 2 \leq x
\end{array}\right.
$$


and

$$
\begin{array}{r}
G_{h}-\frac{b_{h}}{2}>0, \\
G_{h}+\frac{b_{h}}{2}<L, \\
d_{h} \geq \quad-\min _{x \in[0, L]} k_{0}(x), \\
b_{h}<L .
\end{array}
$$

The $2 H$ scalar quantities $b_{h}$ and $d_{h}$ represent the unknowns of the identification procedure.

Introducing in (13) the change of coordinates:

$$
\left\{\begin{array}{c}
d_{h}=\sqrt{A_{h} r_{h}} \\
b_{h}=\sqrt{A_{h} / r_{h}}
\end{array}\right.
$$

each damage width and depth can be expressed through its intensity $A_{h}$ and shape $r_{h}$; the unknowns vector becomes:

$$
\pi=\hat{\pi}(\mathbf{A}, \mathbf{r}) .
$$

With this parametrization, it can be show that, for moderate damage intensities, the sensitivity of the frequency response function (11) with respect to the damage shapes is vanishing, that is:

$$
\lim _{\|\mathbf{A}\|_{\infty} \rightarrow 0} \frac{\left\|\frac{\partial}{\partial \mathbf{r}} \mathfrak{H}(\mathbf{A}, \mathbf{r})\right\|_{\infty}}{\left\|\frac{\partial}{\partial \mathbf{A}} \mathfrak{H}(\mathbf{A}, \mathbf{r})\right\|_{\infty}}=0 .
$$

therefore the coordinates $\mathbf{r}$ can be exclude from the resulting expansion since not observable quantities (see the appendix for an extended proof).

Within this context an identification procedure is reduced to the choice of a suitable functional to be minimized on the space of damage unknowns $\mathbf{A}$ :

$$
\begin{aligned}
& \min _{\alpha \in \mathbb{R}^{+}} F(\alpha, \mathbf{a}) \\
& \|\mathbf{a}\|=1
\end{aligned}
$$

where

$$
\alpha:=\|\mathbf{A}\|, \quad \mathbf{A}=\alpha \mathbf{a}, \quad\|\mathbf{a}\|=1,
$$

and $F$ is an appropriate functional.

Common choices for the functional are either based on difference of eigenfrequencies either of eigenvectors ([2] - [4]). In the first case a suitable functional may be:

$$
F(\alpha, \mathbf{a})=\left\|\varpi(\alpha, \mathbf{a})-\varpi^{0}\right\|
$$


where $\varpi(\alpha, \mathbf{a})=\left\{\varpi_{1}^{*}, \ldots, \varpi_{N}^{*}\right\}$ are the first $N$ mechanical eigenfrequencies of the damaged system, whilst $\varpi^{0}$ the initial state ones.

With the intent of parameters estimation it is more appropriate to introduce the following modified functional:

$$
\begin{aligned}
\widetilde{F}(\alpha, \mathbf{a}) & =\left\|\widehat{\varpi}^{*}-\varpi(\alpha, \mathbf{a})\right\|= \\
& =\left\|\widehat{\varpi}^{*}-\widehat{\varpi}^{0}+\varpi^{0}-\varpi(\alpha, \mathbf{a})\right\| \leq\left\|\widehat{\varpi}^{*}-\widehat{\varpi}^{0}\right\|+\left\|\varpi(\alpha, \mathbf{a})-\varpi^{0}\right\|
\end{aligned}
$$

where the measured eigenfrequencies $\widehat{\varpi}^{*}$ and $\widehat{\varpi}^{0}$ in the damaged and initial state have been introduced.

In (18) it has been tacitly assumed that $\widehat{\varpi}^{0}=\varpi^{0}$, i.e. the model is perfectly tuned to the system in its inital state.

Since $\varpi(\alpha, \mathbf{a})$ are monotonic function of local stiffness ([1]), the functional (18) is convex in each parameter $A_{h}$.

On the opposite FRF functionals, even they are easily tunable and hence suitable to perform sensitivity enhancement techniques, are generally not convex in the damage intensities $A_{h}$. In the next sections we will focus localization method based on eigenfrequencies while the problem of sensitivity enhancement will be addressed in a future work.

\subsection{Model independent pre-localization technique}

In this section we examine the possibility of estimating the damage direction a in a way that is as much as possible independent on the structural model.

To this end a spatial sampling of the FRF function $\tilde{h}_{i j}$ from all the $H$ PZT patches is used to extract information about the change of eigenvectors.

A weighted average of the eigenvectors variations leads to assign at each macroregion a damage probability or in other words a normalized damage intensity.

The effectiveness of the proposed approach strongly relies on the following assumptions:

- large eigenevectors differences are localized in macro-regions subjected to damages;

- the PZT pathces are uniformly distributed to cover all the structural members under observation.

In particular in the next we suppose that the FRF $\mathfrak{H}_{i j}$ has been measured in the frequency range $I=\left[\omega_{i}, \omega_{f}\right]$ in both the damaged and the initial state; such interval satisfies the inequalities:

$$
\omega_{i} \leq \min \left\{\varpi_{1}^{0}, \varpi_{1}^{*}\right\}, \omega_{f} \leq \max \left\{\varpi_{M}^{0}, \varpi_{M}^{*}\right\}
$$

and it can be partitioned as:

$$
I=\bigcup_{m=1}^{M} I_{m}^{0}=\bigcup_{m=1}^{M} I_{m}^{*}
$$


where $I_{m}^{0}=\left[\omega_{i}^{0(m)}, \omega_{f}^{0(m)}\right]$ and $I_{m}^{*}=\left[\omega_{i}^{*(m)}, \omega_{f}^{*(m)}\right]$ are the sub-intervals obtained taking two consecutive minima surrounding each resonant frequency $\varpi_{m}^{0}$, $\varpi_{m}^{*}$ respectively.

In the hypothesis of low damage intensities, we can suppose modal density unchanged and it results that $\varpi_{m}^{0} \approx \varpi_{m}^{*}$.

The subdivision (19) allow introducing the following function:

$$
\Omega_{k}(g):=\left[\frac{1}{\omega_{f_{k}}-\omega_{i_{k}}} \int_{\omega_{i_{k}}}^{\omega_{f_{k}}} g(\omega) \overline{g(\omega)} d \omega\right]^{\frac{1}{2}}
$$

which permit us to extract the contribution of the k-th eigenvector. More precisely, using the spectral decomposition of the response functions:

$$
\begin{aligned}
\mathfrak{H}^{0}(\omega) & \simeq-\omega^{2} \sum_{m=1}^{M} \frac{z_{0}^{(m)} \otimes z_{0}^{(m)}}{\left[\left(\varpi_{m}^{0}\right)^{2}-\omega^{2}\right]+2 \mathfrak{i} \omega \delta_{m} \varpi_{m}^{0}} \\
\mathfrak{H}^{*}(\omega) & \simeq-\omega^{2} \sum_{m=1}^{M} \frac{z_{*}^{(m)} \otimes z_{*}^{(m)}}{\left[\left(\varpi_{m}^{*}\right)^{2}-\omega^{2}\right]+2 \mathfrak{i} \omega \delta_{m} \varpi_{m}^{*}}
\end{aligned}
$$

together with (20), it results:

$$
\Omega_{k}\left(\mathfrak{H}^{0}\right)=\left[\frac{1}{\omega_{f_{k}}^{0}-\omega_{i_{k}}^{0}} \sum_{m=1}^{M} \int_{\omega_{i_{k}}^{0}}^{\omega_{f_{k}}^{0}} \omega^{4} \frac{z_{0}^{(m)} \otimes z_{0}^{(m)}}{\left(-\omega^{2}+\varpi_{m}^{2}\right)^{2}+4 \omega^{2} \delta_{h}^{2} \varpi_{m}^{2}} d \omega\right]^{\frac{1}{2}}
$$

since $\left\{z_{0}^{(1)}, z_{0}^{(2)}, \ldots, z_{0}^{(M)}\right\}$ are a set of orthonormal eigenvectors.

From the mean theorem for each $k$ there exists a frequency $\omega_{\xi_{k}} \in\left[\omega_{i_{k}}, \omega_{f_{k}}\right]$, such that:

$$
\Omega_{k}\left(\mathfrak{H}^{0}\right)=\left[\frac{1}{\omega_{f_{k}}^{0}-\omega_{i_{k}}^{0}} \sum_{m=1}^{M} \omega_{\xi_{k}}^{4} \frac{z_{0}^{(m)} \otimes z_{0}^{(m)}}{\left(-\omega_{\xi_{k}}^{2}+\varpi_{m}^{2}\right)^{2}+4 \omega_{\xi_{k}}^{2} \delta_{m}^{2} \varpi_{m}^{2}}\right]^{\frac{1}{2}}
$$

and in the case of low modal density, the following approximation holds:

$$
\Omega_{k}\left(\mathfrak{H}^{0}\right) \simeq\left[\frac{\omega_{\xi_{k}}^{4}}{\omega_{f_{k}}^{0}-\omega_{i_{k}}^{0}} \frac{z_{0}^{(k)} \otimes z_{0}^{(k)}}{\left(-\omega_{\xi_{k}}^{2}+\varpi_{k}^{2}\right)^{2}+4 \omega_{\xi_{k}}^{2} \delta_{k}^{2} \varpi_{k}^{2}}\right]^{\frac{1}{2}}
$$

Equation ((24) show that the introduced functional $\Omega_{k}$ effectively selects the modal shape $z_{0}^{(k)}$ associated to $k$-th frequency interval.

Once each eigenvector in the frequency range $I$ has been extracted from both $\mathfrak{H}^{0}$ and $\mathfrak{H}^{*}$, the following weighted average:

$$
\nu=\frac{\tilde{\nu}}{\|\tilde{\nu}\|_{2}}, \quad \tilde{\nu}=\frac{1}{M} \sum_{m=1}^{M}\left(z_{*}^{(m)}-z_{0}^{(m)}\right)
$$




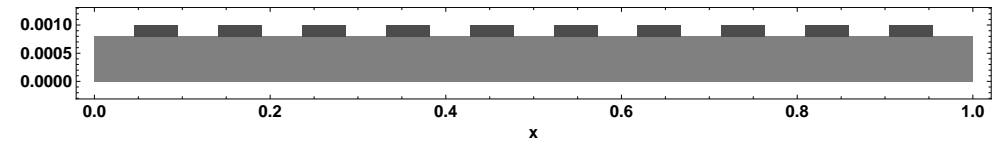

Figure 1: Electromechanical beam composed by 15-th piezoelectric patches.

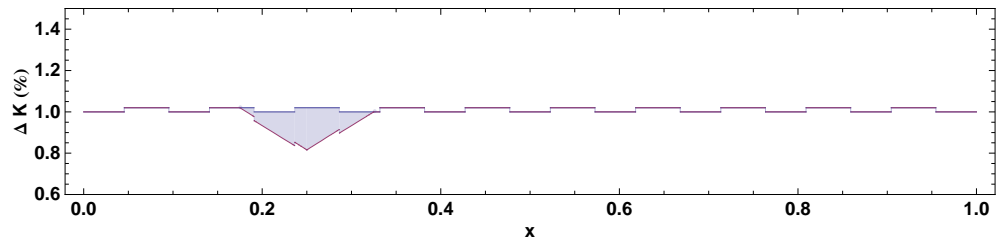

Figure 2: Stiffness variation (percentage) along the beam in the case of single damage.

permit us to assign to each macro-region a scalar coefficient that represents a normalized stiffness variation in that sub-region.

We remark here that the eigenvectors difference $(25)$ from the function $\mathfrak{H}$ in a model independent way (in the hypothesis on PZT distribution clarified at start of this section).

This estimation allow us to reduce the localization problem to:

$$
\min _{\alpha \in \mathbb{R}^{+}} F\left(\alpha, \mathbf{a}_{P}\right)
$$

which can be solved with standard minimization techniques.

\section{Numerical Examples}

The effectiveness of the proposed method will be proved on a numerical problem concerning the system represented in figure (1): it consists of a cantilever beam with 10 PZT patches.

The cases of single and multiple damages occurring in the positions showed in figure (2) - (3) have been examined and the result of the pre-localization technique are shown in next figures.

In particular figures (4) - (7) represent the first two eigenvectors of the system in the initial state and after the damage has occurred. They allow to calculate through equation (25) the normalizes stiffness variation showed in the figures (4) - (4) for the case of single and double damage respectively.

The reduced minimization problem (26) has been solved with the help of Mathematica minimization routine based on Quasi-Newton method and the results are showed in figure (10). 


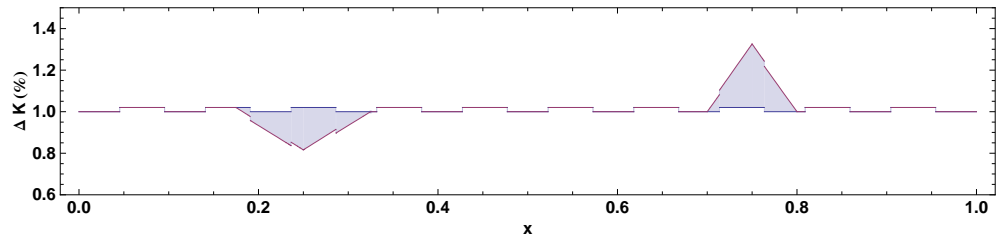

Figure 3: Stiffness variation (percentage) along the beam in the case of multiple damages.

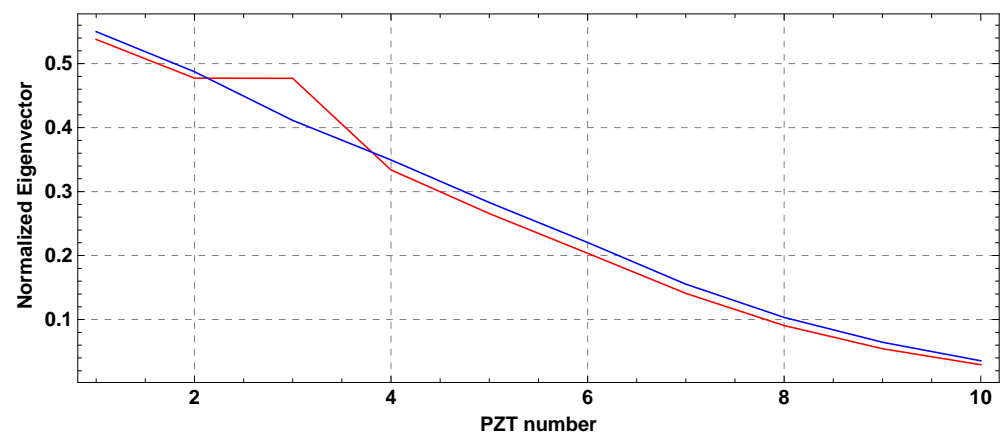

Figure 4: First eigenvector of the FRF extracted from the $\mathfrak{H}^{0}$ (blue) and $\mathfrak{H}^{*}$ (red) in the case of single damage.

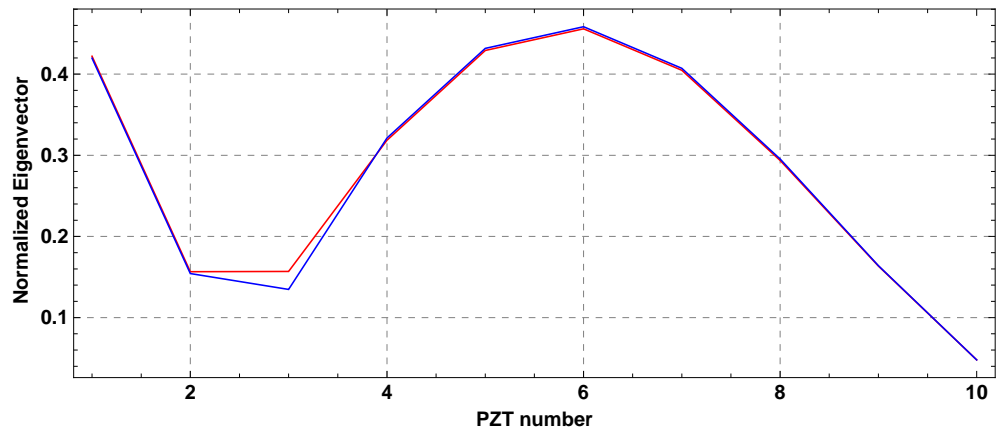

Figure 5: Second eigenvector of the FRF extracted from the $\mathfrak{H}^{0}$ (blue) and $\mathfrak{H}^{*}$ (red)in the case of single damage. 


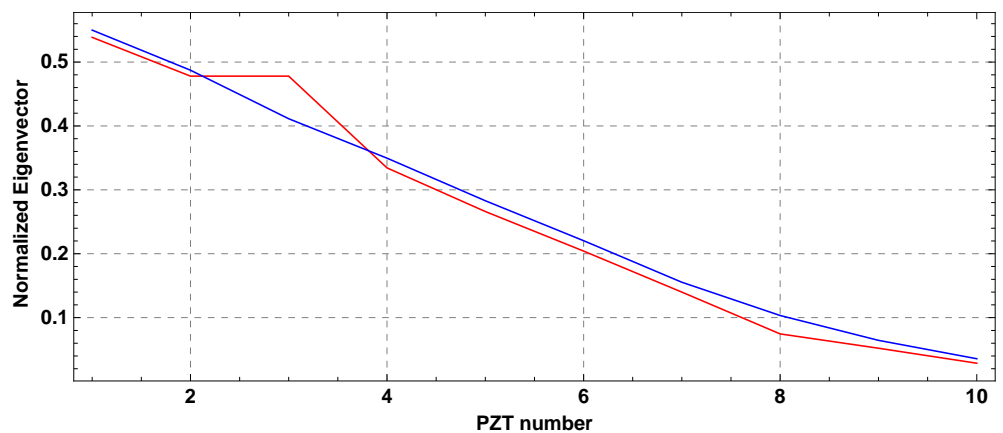

Figure 6: First eigenvector of the FRF extracted from the $\mathfrak{H}^{0}$ (blue) and $\mathfrak{H}^{*}$ (red).

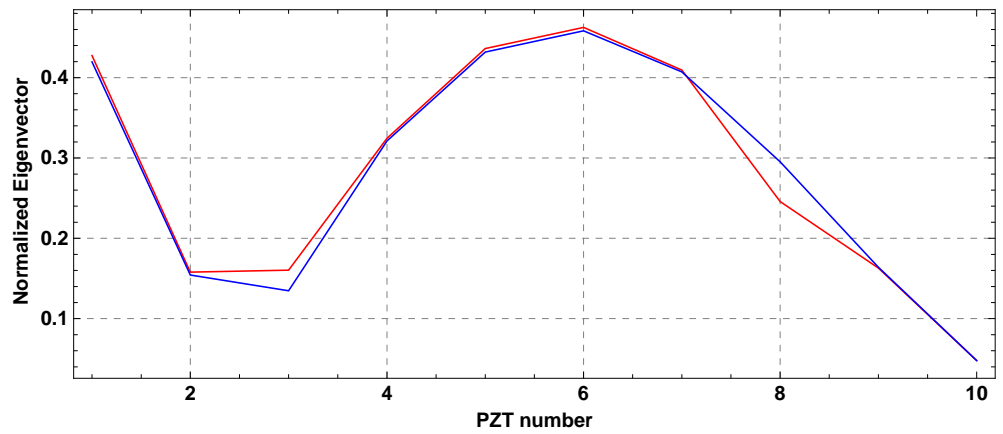

Figure 7: Second eigenvector of the FRF extracted from the $\mathfrak{H}^{0}$ (blue) and $\mathfrak{H}^{*}$ (red).

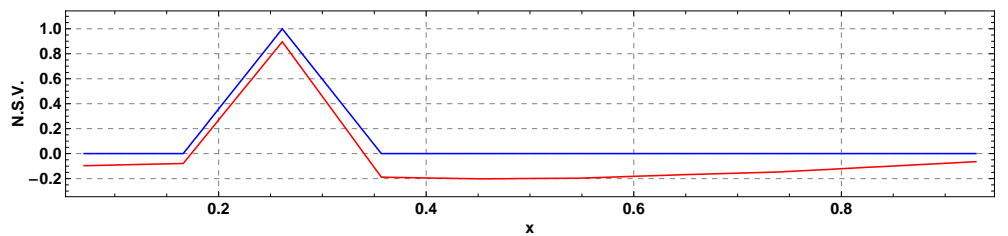

Figure 8: Normalized Stiffness Variation (estimated - red, actual - blue).

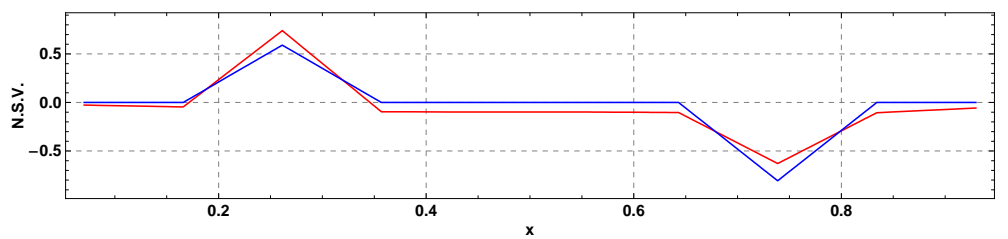

Figure 9: Normalized Stiffness Variation (estimated - red, actual - blue). 


\begin{tabular}{|c|cc|c|c|c|c|}
\hline Damages Num. & \multicolumn{2}{|c|}{ Actual Param. } & \multicolumn{2}{|c|}{ Estimated Param. } & \multicolumn{2}{c|}{ Relative Error } \\
\hline & Position & Area (A) & Position & Area $(A)$ & Positon & Area (A) \\
\hline & & & & & & \\
\hline & 0.2500 & 0.0150 & 0.2590 & 0.0159 & 0.0360 & 0.0600 \\
\hline $\mathbf{2}$ & 0.2500 & 0.0150 & 0.2590 & 0.0158 & 0.0360 & 0.0530 \\
\hline & 0.7500 & -0.0150 & 0.7460 & -0.0146 & -0.0050 & -0.0260 \\
\hline
\end{tabular}

Figure 10: Results of the identification procedure on the case of study.

\section{Conclusions}

The damage detection method presented in this work is aimed to estimate both damage positions and intensities. The localization is as much as possible independent on the structural model and it is based on the change of the eigenvectors of the Frequency Response Function ( $F R F)$.

This procedure has the undoubted advantage to be easily measurable since it reacquires the acquisition of the impedance matrix $\mathbf{N}$ through a simple electric circuit.

Once the damages have been localized the estimate of their intensities reduces to a minimization problem with can be solved with standard technique.

Easily measurability and model Independence clearly demonstrate applicability of the proposed method to real structures.

\section{A Proof of main results}

\section{A.1 Independence of the damage functional on the shape}

Equation (11) can be rewritten in terms of the $\mathbf{r}$ and $\mathbf{A}$ variables as:

$$
\mathfrak{H}_{i j}(\mathbf{A}, \mathbf{r}):=s^{2} \Gamma_{\alpha i} H_{\alpha \beta}(\mathbf{A}, \mathbf{r}) \Gamma_{\beta j},
$$

which by differentiation becomes:

$$
\begin{aligned}
\frac{\partial \mathfrak{H}_{i j}(\mathbf{A}, \mathbf{r})}{\partial r_{h}} & =\frac{\partial \mathfrak{H}_{i j}}{\partial b_{k}} \frac{\partial b_{k}}{\partial r_{h}}+\frac{\partial \mathfrak{H}_{i j}}{\partial d_{k}} \frac{\partial d_{k}}{\partial r_{h}} \\
\frac{\partial \mathfrak{H}_{i j}(\mathbf{A}, \mathbf{r})}{\partial A_{h}} & =\frac{\partial \mathfrak{H}_{i j}}{\partial b_{k}} \frac{\partial b_{k}}{\partial A_{h}}+\frac{\partial \mathfrak{H}_{i j}}{\partial d_{k}} \frac{\partial d_{k}}{\partial A_{h}}
\end{aligned}
$$

The gradient $C_{i j k}^{0}$ of the function $\mathfrak{H}_{i j}(\pi)$ with respect to the parameters near $\pi=\pi_{0}$ is easily obtained using the definitions of $\mathfrak{H}$ and $H$ :

$$
\begin{aligned}
C_{i j k}^{0} & =\left.\frac{\partial \mathfrak{H}_{i j}(\pi)}{\partial \pi_{p}}\right|_{\pi=\pi_{0}}=-\left.s^{2} \Gamma_{\alpha i} \frac{\partial H_{\alpha \beta}(\pi)}{\partial \pi_{p}}\right|_{\pi=\pi_{0}} \Gamma_{\beta j}= \\
& =\left.s^{2} \Gamma_{\alpha i} H_{\alpha \gamma}\left(\pi_{0}\right) \frac{\partial K_{\gamma \delta}(\pi)}{\partial \pi_{p}}\right|_{\pi=\pi_{0}} H_{\delta \beta}\left(\pi_{0}\right) \Gamma_{\beta j}
\end{aligned}
$$


hence in terms of damage width and height:

$$
\begin{aligned}
& \frac{\partial \mathfrak{H}_{i j}(\mathbf{A}, \mathbf{r})}{\partial b_{k}}=s^{2} \Gamma_{\alpha i} H_{\alpha \gamma}\left(\pi_{0}\right) \frac{\partial K_{\gamma \delta}(\pi)}{\partial b_{k}} H_{\delta \beta}\left(\pi_{0}\right) \Gamma_{\beta j} ; \\
& \frac{\partial \mathfrak{H}_{i j}(\mathbf{A}, \mathbf{r})}{\partial d_{k}}=s^{2} \Gamma_{\alpha i} H_{\alpha \gamma}\left(\pi_{0}\right) \frac{\partial K_{\gamma \delta}(\pi)}{\partial d_{k}} H_{\delta \beta}\left(\pi_{0}\right) \Gamma_{\beta j} .
\end{aligned}
$$

Since $\Delta K_{\gamma \delta}(\pi)=K_{\gamma \delta}(\pi)-K_{\gamma \delta}\left(\pi_{0}\right)$ and $\phi_{\rho}^{\prime \prime}$ are smooth functions of integration variable $x$, the following inequality holds

$$
\begin{aligned}
\left|\Delta K_{\gamma \delta}\left(d_{k}, b_{k} ; x\right)\right| & =\left|\int_{0}^{l} d_{k} f_{k}\left(b_{k} ; x\right) \phi_{\gamma}^{\prime \prime}(x) \phi_{\delta}^{\prime \prime}(x) d x\right| \\
& \leq\left|d_{k}\right|\left|b_{k}\right| \max _{x \in F_{k}}\left|\phi_{\gamma}^{\prime \prime}(x) \phi_{\delta}^{\prime \prime}(x)\right| \\
& \leq c\left|b_{k}\right|\left|d_{k}\right|
\end{aligned}
$$

being $F_{k}=\left[G_{k}-\frac{b_{k}}{2}, G_{k}+\frac{b_{k}}{2}\right]$ and $c=\max _{x \in F_{k}}\left|\phi_{\gamma}^{\prime \prime}(x) \phi_{\delta}^{\prime \prime}(x)\right|$.

For $d_{k} \rightarrow 0$ and $b_{k} \rightarrow 0$ respectively, one gets

$$
\mid \begin{aligned}
& \left|\frac{\partial K_{\gamma \delta}(\xi, \mathbf{d}, \mathbf{b} ; x)}{\partial d_{k}}\right| \leq c b_{k} \\
& \left|\frac{\partial K_{\gamma \delta}(\xi, \mathbf{d}, \mathbf{b} ; x)}{\partial b_{k}}\right| \leq c d_{k}
\end{aligned}
$$

hence

$$
\begin{aligned}
& \left\|\frac{\partial \mathbf{K}}{\partial \mathbf{b}}\right\|_{\infty} \leq c\|\mathbf{d}\|_{\infty} \\
& \left\|\frac{\partial \mathbf{K}}{\partial \mathbf{d}}\right\|_{\infty} \leq c\|\mathbf{b}\|_{\infty}
\end{aligned}
$$

In addition, from equation (14), the following holds:

$$
\begin{aligned}
& \left|\frac{\partial b_{h}}{\partial r_{k}}\right|=\delta_{k}^{h} \frac{\sqrt{A_{h}}}{2 \sqrt{r_{h}^{3}}} ; \\
& \left|\frac{\partial b_{h}}{\partial A_{k}}\right|=\delta_{k}^{h} \frac{1}{2 \sqrt{A_{h} r_{h}}} ; \\
& \left|\frac{\partial d_{h}}{\partial r_{k}}\right|=\delta_{k}^{h} \frac{\sqrt{A_{h}}}{2 \sqrt{r_{h}}} ; \\
& \left|\frac{\partial d_{h}}{\partial A_{k}}\right|=\delta_{k}^{h} \frac{1}{2 \sqrt{r_{h}^{3}} \sqrt{A_{h}}} ;
\end{aligned}
$$


where we have used the Kronecker delta $\delta_{k}^{h}$. Introducing the notation:

$$
\begin{aligned}
\left\|\frac{\partial \mathbf{b}}{\partial \mathbf{r}}\right\|_{\infty} & =\sup _{h=1, \ldots, N}\left\{\frac{\sqrt{A_{h}}}{2 \sqrt{r_{h}^{3}}}\right\}, \\
\left\|\frac{1}{2 \sqrt{\mathbf{A}} \sqrt{\mathbf{r}}}\right\|_{\infty} & =\sup _{h=1, \ldots, N}\left\{\frac{1}{2 \sqrt{A_{h}} \sqrt{r_{h}}}\right\}, \\
\left\|\frac{\sqrt{\mathbf{A}}}{2 \sqrt{\mathbf{r}}}\right\|_{\infty} & =\sup _{h=1, \ldots, N}\left\{\frac{\sqrt{A_{h}}}{2 \sqrt{r_{h}}}\right\}, \\
\left\|\frac{1}{2 \sqrt{\mathbf{r}^{3}} \sqrt{\mathbf{A}}}\right\|_{\infty} & =\sup _{h=1, \ldots, N}\left\{\frac{1}{2 \sqrt{r_{h}} \sqrt{A_{h}}}\right\},
\end{aligned}
$$

the following inequalities hold:

$$
\begin{aligned}
&\left\|\frac{\partial \mathbf{b}}{\partial \mathbf{r}}\right\|_{\infty} \leq\left\|\frac{\sqrt{\mathbf{A}}}{2 \sqrt{\mathbf{r}^{3}}}\right\|_{\infty} ; \\
&\left\|\frac{\partial \mathbf{b}}{\partial \mathbf{A}}\right\|_{\infty} \leq\left\|\frac{1}{2 \sqrt{\mathbf{A}} \sqrt{\mathbf{r}}}\right\|_{\infty} ; \\
&\left\|\frac{\partial \mathbf{d}}{\partial \mathbf{r}}\right\|_{\infty} \leq\left\|\frac{\sqrt{\mathbf{A}}}{2 \sqrt{\mathbf{r}}}\right\|_{\infty} ; \\
&\left\|\frac{\partial \mathbf{d}}{\partial \mathbf{A}}\right\|_{\infty} \leq\left\|\frac{1}{2 \sqrt{\mathbf{r}^{3}} \sqrt{\mathbf{A}}}\right\|_{\infty} .
\end{aligned}
$$

Previous equations along with (28) establish an upper bound for the sensitivity of the functional with respect to damage shape and intensity:

$$
\begin{aligned}
& \left\|\frac{\partial \mathfrak{H}(\mathbf{A}, \mathbf{r} ; x)}{\partial \mathbf{r}}\right\|_{\infty} \leq c\|\mathbf{d}\|_{\infty}\left\|\frac{\sqrt{\mathbf{A}}}{2 \sqrt{\mathbf{r}^{3}}}\right\|_{\infty}+c\|\mathbf{b}\|_{\infty}\left\|\frac{\sqrt{\mathbf{A}}}{2 \sqrt{\mathbf{r}}}\right\|_{\infty}, \\
& \left\|\frac{\partial \mathfrak{H}(\mathbf{A}, \mathbf{r} ; x)}{\partial \mathbf{A}}\right\|_{\infty} \leq c\|\mathbf{d}\|_{\infty}\left\|\frac{1}{2 \sqrt{\mathbf{A}} \sqrt{\mathbf{r}}}\right\|_{\infty}+c\|\mathbf{b}\|_{\infty}\left\|\frac{1}{2 \sqrt{\mathbf{r}^{3}} \sqrt{\mathbf{A}}}\right\|_{\infty},
\end{aligned}
$$

It results

$\lim _{\|A\|_{\infty} \rightarrow 0} \frac{\left\|\frac{\partial \mathfrak{H}(\mathbf{A}, \mathbf{r} ; x)}{\partial \mathbf{r}}\right\|_{\infty}}{\left\|\frac{\partial \mathfrak{H}(\mathbf{A}, \mathbf{r} ; x)}{\partial \mathbf{A}}\right\|_{\infty}} \leq \lim _{\|A\|_{\infty} \rightarrow 0} \frac{\|\mathbf{d}\|_{\infty}\left\|\frac{\sqrt{\mathbf{A}}}{2 \sqrt{\mathbf{r}^{3}}}\right\|_{\infty}+\|\mathbf{b}\|_{\infty}\left\|\frac{\sqrt{\mathbf{A}}}{2 \sqrt{\mathbf{r}}}\right\|_{\infty}}{\|\mathbf{d}\|_{\infty}\left\|\frac{1}{2 \sqrt{\mathbf{A}} \sqrt{\mathbf{r}}}\right\|_{\infty}+\|\mathbf{b}\|_{\infty}\left\|\frac{1}{2 \sqrt{\mathbf{r}^{3}} \sqrt{\mathbf{A}}}\right\|_{\infty}}=0$

that is for small damage intensities the sensitivity of the response function $\mathfrak{H}$ is vanishing with respect to the damage shape. 


\section{References}

[1] V. I. Arnold. Mathematical Methods of Classical Mechanics. Springer, 1981.

[2] D. Bernarl. Load vectors for damage localization. Journal of Engineering Mechanics, (128(1)), 2002.

[3] F. dell'Isola, C. Maurini, and M. Porfiri. Passive damping of beam vibrations through distributed electric networks and piezoelectric transducers: prototype design and experimental validation. Smart Materials and Structures, (13):299-308, 2004.

[4] J. K. Sinha and M. I. Friswell. The location of spring supports from measured vibration data. Journal of Sound and Vibrations, (244(1)):137-153, 2001. 